\title{
EchoGéo
}

15 | 2011

La Belgique, État prospère en décomposition au cœur de l'Europe du nord-ouest

\section{Une géographie de la coopération universitaire France-Brésil, analyse des accords Capes-Cofecub}

\section{Hervé Théry}

\section{(2) OpenEdition}

\section{Journals}

Édition électronique

URL : https://journals.openedition.org/echogeo/12296

DOI : $10.4000 /$ echogeo.12296

ISSN : 1963-1197

Éditeur

Pôle de recherche pour l'organisation et la diffusion de l'information géographique (CNRS UMR 8586)

Référence électronique

Hervé Théry, « Une géographie de la coopération universitaire France-Brésil, analyse des accords

Capes-Cofecub », EchoGéo [En ligne], 15 | 2011, mis en ligne le 11 avril 2011, consulté le 03 août 2021.

URL : http://journals.openedition.org/echogeo/12296 ; DOI : https://doi.org/10.4000/echogeo.12296

Ce document a été généré automatiquement le 3 août 2021.

EchoGéo est mis à disposition selon les termes de la licence Creative Commons Attribution - Pas d'Utilisation Commerciale - Pas de Modification 4.0 International (CC BY-NC-ND) 


\title{
Une géographie de la coopération universitaire France-Brésil, analyse des accords Capes-Cofecub
}

\author{
Hervé Théry
}

\section{NOTE DE L'ÉDITEUR}

Le manuscrit initial de cet article a été soumis en novembre 2009

1 La coopération scientifique franco-brésilienne a déjà une longue histoire (Martinière 1982), dont l'un des épisodes marquants a été la participation d'une forte mission française à la fondation de l'Universidade de São Paulo (USP) en 1934 (Lefèvbre 1990). Parmi les jeunes professeurs qui la composaient et qui ont pour la plupart fait ensuite de belles carrières et largement contribué au renouveau de leurs domaines (Claude Lévi-Strauss, Roger Bastide, Fernand Braudel) figurait Pierre Monbeig, qui a eu un rôle décisif dans le développement de la géographie scientifique au Brésil et dans la naissance de l'AGB, l'association des géographes brésiliens.

Cette collaboration s'est prolongée depuis lors, avec des hauts et des bas inévitables, (notamment le recul de l'influence française au profit de celle des États-Unis dans certains domaines), mais dans d'autres champs, comme les sciences sociales, elle a pu se maintenir à un haut niveau et des mécanismes ont été mis en place pour favoriser les échanges. Parmi ces instruments, un rôle essentiel a été tenu par les accords dits "Capes-Cofecub », du nom des deux organismes qui le gèrent, la Coordination pour le Perfectionnement $d u$ Personnel de l'Enseignement Supérieur du Ministère de l'Éducation brésilienne (à peu de choses près sa Direction de l'Enseignement Supérieur) et le Comité Français d'Évaluation de la Coopération Universitaire et Scientifique avec le Brésil, petite structure ad hoc créée pour évaluer et piloter les programmes de coopération en matière de recherche et de formation avec le Brésil. 
3 Ces accords méritent une étude spécifique, doublement géographique. D’abord en ce qu'elle prête une spéciale attention à la localisation des projets en France et au Brésil, aux échanges qui se configurent entre des villes des deux pays, situant la coopération franco-brésilienne dans la géographie des grands pôles scientifiques des deux pays. Ensuite parce qu'elle se focalise, dans sa dernière partie, sur les projets conclus entre départements de géographie des Universités françaises et brésiliennes. Les données disponibles sur les sites du Capes et du Cofecub ont été utilisées pour une étude statistique et cartographique qui récapitule et cartographie les 750 projets approuvés et exécutés au cours de ces trente ans.

\section{Principes et modalités de l'accord Capes-Cofecub}

4 Cet accord cadre de coopération interuniversitaire, signé en 1979, a pour but de pérenniser les échanges de haut niveau entre les deux pays. La Capes cofinance à part égale les missions courtes des chercheurs et universitaires et finance en totalité les boursiers doctorants brésiliens. Le programme Capes-Cofecub a permis d'organiser 524 missions en 2007, de lancer au 1er janvier 200937 nouveaux projets, sur un total de 120 projets en cours (16 ayant commencé en 2006, 33 en 2007 et 34 en 2008). Du côté français, la tutelle (et le financement) sont assurés par le ministère des Affaires étrangères et européennes et le ministère de l'Enseignement supérieur et de la Recherche.

Un séminaire commémore 30 ans de coopération franco-brésilienne

En trois décennies, l'accord de coopération universitaire entre le Brésil et la France, dit Capes/Cofecub, a aidé
à développer 659 projets dans tous les champs disciplinaires et, actuellement, environ 1600 étudiants
brésiliens développent des projets en France [...] Le président du Cofecub, M. Pierre Jaisson, attribue ce succès
aux affinités qui existent entre la France et le Brésil, principalement dans le champ des initiatives
scientifiques. «Il y a un sentiment d'identité et une complémentarité entre les deux pays. Le Brésil est [...] à la
hauteur de la France, avec son dynamisme, son développement extrêmement rapide et sa puissance de
recherche ", a-t-il remarqué. "Le Capes/Cofecub est la base, le sol sur lequel reposent d'autres projets de
coopération, ce qui permet de renouveler la recherche entre les deux pays ", a affirmé Pierre Colombier,
conseiller de Coopération et Action Culturelle de l'Ambassade de la France au Brésil. "C'est la matrice qui fait
apparaître une nouvelle génération de chercheurs", a-t-il ajouté. À son tour, le président de la Capes, Jorge
Guimarães, classe le début du programme comme "modeste et asymétrique ", puisque c'étaient surtout les
Brésiliens qui allaient en France. Il voit dans ce qui arrive aujourd'hui un "moment plus symétrique ", et se
réjouit de l'équilibre qui s'est installé dans les échanges de chercheurs entre les deux pays. Il a indiqué que le
nombre des boursiers brésiliens qui choisissent d'étudier en France, par le biais du programme de
coopération, dépasse ceux qui ont eu pour destination les États-Unis. L'année dernière, 1192 étudiants
brésiliens ont reçu des bourses d'étude pour aller en France, contre 946 pour les États-Unis.

Source : Article publié le 25 mai 2009 sur le site de l'Année de la France au Brésil.

5 Le programme est ouvert à tous les champs disciplinaires ${ }^{1}$, chaque projet devant permettre une collaboration sur un projet scientifique commun afin de former des docteurs brésiliens. Le responsable du projet doit être obligatoirement en activité, publiant et habilité à diriger des recherches au sein d'un laboratoire reconnu et ne peut diriger qu'un seul projet Cofecub.La sélection des projets est réalisée sur la base de 
l'excellence, indépendamment du champ disciplinaire, les propositions sont évaluées en parallèle par des experts anonymes désignés par le Cofecub et par la Capes, les résultats des deux comités sont ensuite confrontés lors d'une réunion décisionnelle commune.

Les projets sont retenus pour une durée de 4 ans, sauf avis contraire des comités français et brésilien à l'issue de la deuxième année.Le programme fournit un appui pour des missions liées au développement du projet pour des chercheurs confirmés. Il assure également les frais de séjours pour des stages doctoraux ou postdoctoraux (d'une durée de deux mois pour les doctorants français).

7 Côté français, le Cofecub prend en charge les frais de transport pour les missions vers le Brésil et ceux de séjour pour les Brésiliens et attribue dans certains cas des stages d'une durée de 2 mois pour des étudiants français se rendant au Brésil.Côté brésilien, la Capes prend en charge le transport des chercheurs, doctorants et post-doctorants brésiliens ainsi que les indemnités de séjours des Français. Elle attribue également deux bourses par an pour des stages doctoraux (4 à 12 mois), des stages doctoraux en cotutelle (4 à 18 mois) et des stages postdoctoraux (2 à 12 mois).

\section{Participants, évolution et thèmes}

La répartition des universités participantes de part et d'autre de l'Atlantique (figure 1) n'est évidemment pas sans rapport avec la hiérarchie urbaine dans les deux pays, et l'on n'est pas surpris de constater que Paris, dans le cas français, et Rio et São Paulo au Brésil se détachent nettement du reste des villes du pays. On constate toutefois la présence de villes de taille plus petite, par exemple Londrina, Maringá (Paraná) ou Feira de Santana (Bahia) au Brésil, ainsi que le Mans ou Concarneau, du côté français.

Figure 1 - Les Universités participant à l'accord Capes-Cofecub

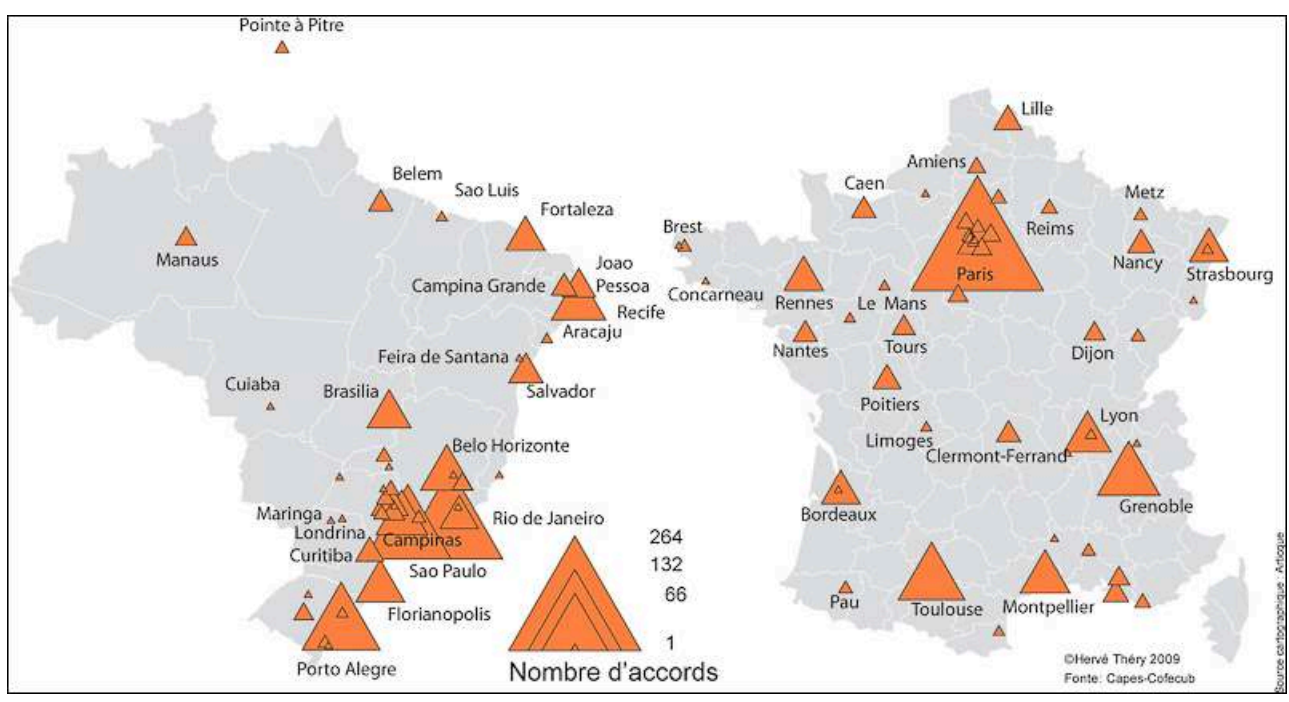

Source : Capes-Cofecub - H. Théry, 2009.

9 L'évolution dans le temps des accords (figure 2) fait apparaître une augmentation de la part des grandes capitales avec le passage des décennies: alors que Toulouse, par exemple se détachait nettement dans les années 70 et 80 , sa place se réduit au fil du 
temps, alors que celle de Paris augmente, et symétriquement, la part de Fortaleza ou Belém diminue, au profit des grandes métropoles du Sudeste.

On peut expliquer cette évolution par le fait qu'à l'origine le programme visait à renforcer le potentiel des universités du Nord et du Nordeste, une ambition de rééquilibrage géographique qui a progressivement cédé la place à la collaboration entre universités d'excellence, sans forcément prendre en compte ce souci de rééquilibrage du territoire.

Figure 2 - Évolution par décennie

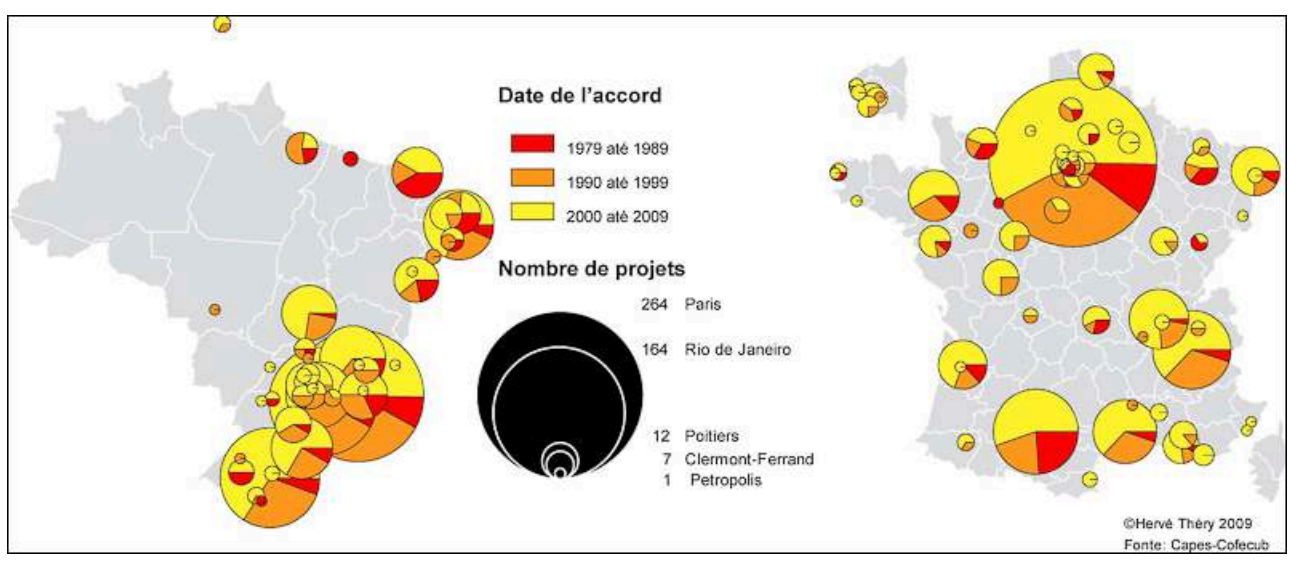

Source : Capes-Cofecub - H. Théry, 2008.

11 En terme de répartition par champs scientifiques, la prépondérance des sciences exactes et de la Terre, suivies par les sciences de l'ingénieur est manifeste puisqu'elles représentent à elles deux la moitié des projets appuyés au cours de ces 30 ans. Les sciences humaines et la biologie ne les suivent que d'assez loin, l'agronomie et la santé fermant le cortège avec des pourcentages inférieurs à $10 \%$ du total.

Tableau 1 - Champs scientifiques

\begin{tabular}{|l|l|l|}
\hline & Nombre de projets & $\%$ du total \\
\hline Sciences exactes et de la Terre & 242 & 32 \\
\hline Sciences pour l'ingénieur & 140 & 18 \\
\hline Sciences humaines & 103 & 13 \\
\hline Biologie & 101 & 13 \\
\hline Sciences sociales appliquées & 57 & 7 \\
\hline Agronomie & 49 & 6 \\
\hline \hline Santé & 37 & 5 \\
\hline \hline Linguistique, lettres et arts & 29 & 4 \\
\hline
\end{tabular}




\begin{tabular}{|l|l|l|}
\hline Multidisciplinaire & 5 & 1 \\
\hline
\end{tabular}

Figure 3 - Champs scientifiques

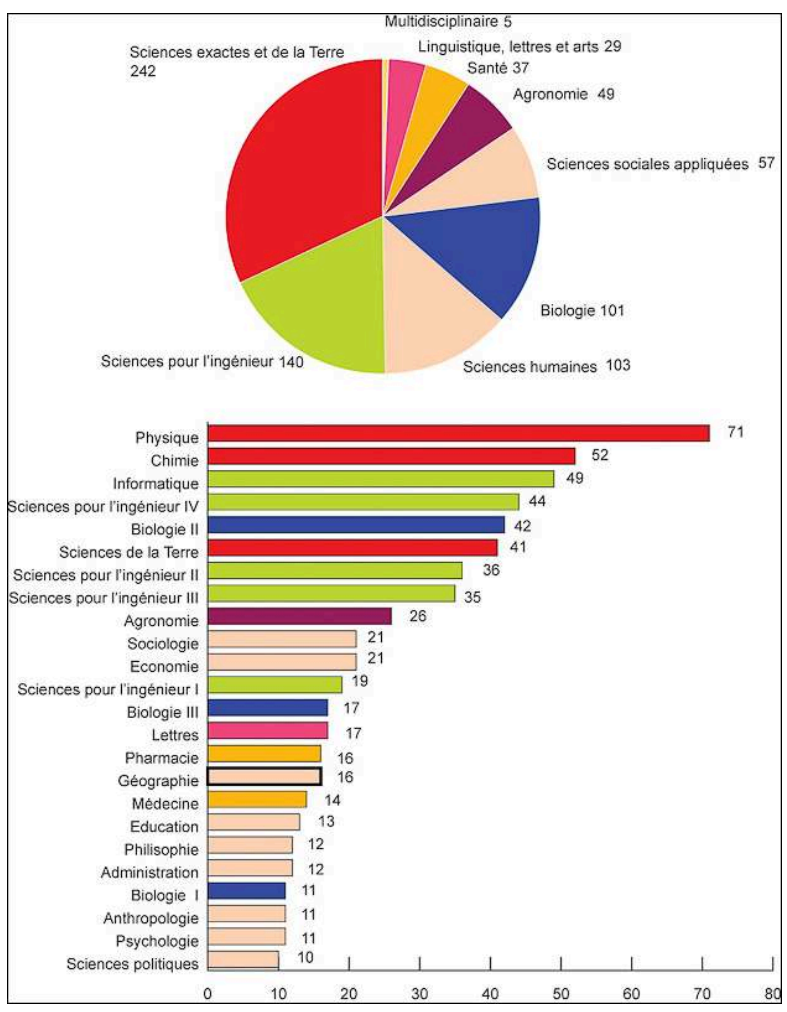

12 Si l'on passe de la répartition globale à une analyse ville par ville, on n'observe pas de spécialisation absolue (à part de rares exceptions près, comme Brest ou Concarneau, Uberaba ou Vitória). Il est très rare que des villes apparaissent sur la figure 3 sous forme d'un cercle d'une seule couleur, ce qui indiquerait qu'elle n'est active - dans le cadre de la coopération franco-brésilienne - que d'une seule aire scientifique. Dans tous les autres cas, les figures en secteur de cercle ${ }^{2}$ représentant les aires scientifiques présentes dans chaque ville montrent un assez bon équilibre entre elles, même dans des villes de taille moyenne. Tout au plus peut-on noter la part importante des sciences de l'ingénieur à Nancy, des sciences exactes à Grenoble, des sciences agraires à Viçosa et Piracicaba, deux centres universitaires à nette dominante agronomique.

Tableau 2 - Champs scientifiques par ville (dix premières villes)

\begin{tabular}{|l|l|l|l|l|l|l|l|l|l|}
\hline & Total & $\begin{array}{l}\text { Sciences } \\
\text { exactes } \\
\text { et de la } \\
\text { Terre }\end{array}$ & $\begin{array}{l}\text { Sciences } \\
\text { pour } \\
\text { l'ingénieur }\end{array}$ & $\begin{array}{l}\text { Sc. } \\
\text { humaines }\end{array}$ & Biologie & $\begin{array}{l}\text { Sc. Soc. } \\
\text { appliquées }\end{array}$ & Agronomie & Santé & $\begin{array}{l}\text { Linguis. } \\
\text { lettres } \\
\text { et arts }\end{array}$ \\
\hline \hline Rio de & 164 & 30 & 45 & 23 & 30 & 23 & 3 & 5 & 4 \\
\hline
\end{tabular}




\begin{tabular}{|c|c|c|c|c|c|c|c|c|c|}
\hline São Paulo & 111 & 42 & 12 & 19 & 16 & 6 & 5 & 3 & 7 \\
\hline Porto Alegre & 92 & 52 & 6 & 15 & 5 & 4 & 3 & 5 & 2 \\
\hline Recife & 45 & 14 & 6 & 5 & 6 & 5 & 1 & 7 & 1 \\
\hline $\begin{array}{l}\text { Belo } \\
\text { Horizonte }\end{array}$ & 42 & 10 & 10 & 4 & 10 & 1 & & 3 & 4 \\
\hline Campinas & 40 & 7 & 8 & 8 & 4 & 2 & 6 & & 5 \\
\hline Florianópolis & 36 & 7 & 16 & 1 & 2 & 4 & 2 & & 4 \\
\hline Brasília & 29 & 8 & 8 & 4 & 6 & 1 & 1 & & \\
\hline Fortaleza & 24 & 7 & 2 & 4 & 7 & 2 & 1 & 1 & \\
\hline Niterói & 22 & 11 & 1 & 8 & 1 & & & & \\
\hline Paris & 351 & 69 & 32 & 52 & 34 & 35 & 12 & 15 & 15 \\
\hline Toulouse & 73 & 21 & 28 & 5 & 4 & 1 & 7 & & 1 \\
\hline Grenoble & 68 & 32 & 10 & 2 & 3 & 7 & & 1 & 4 \\
\hline Montpellier & 44 & 8 & 2 & 4 & 9 & 2 & 9 & 2 & \\
\hline Lyon & 39 & 9 & 12 & 4 & 3 & 1 & & 2 & 2 \\
\hline Strasbourg & 28 & 7 & 2 & 5 & 6 & & & 2 & 1 \\
\hline Rennes & 28 & 12 & 3 & 4 & 2 & & 3 & & \\
\hline Bordeaux & 24 & 11 & 4 & 1 & 4 & & 1 & 2 & \\
\hline $\begin{array}{l}\text { Aix en } \\
\text { Provence }\end{array}$ & 16 & 3 & & 2 & 2 & 3 & & & 1 \\
\hline Caen & 15 & 1 & & 6 & 1 & & 1 & & \\
\hline Poitiers & 13 & 10 & & 1 & & & 1 & & \\
\hline
\end{tabular}


Figure 4 - Champs scientifiques par ville

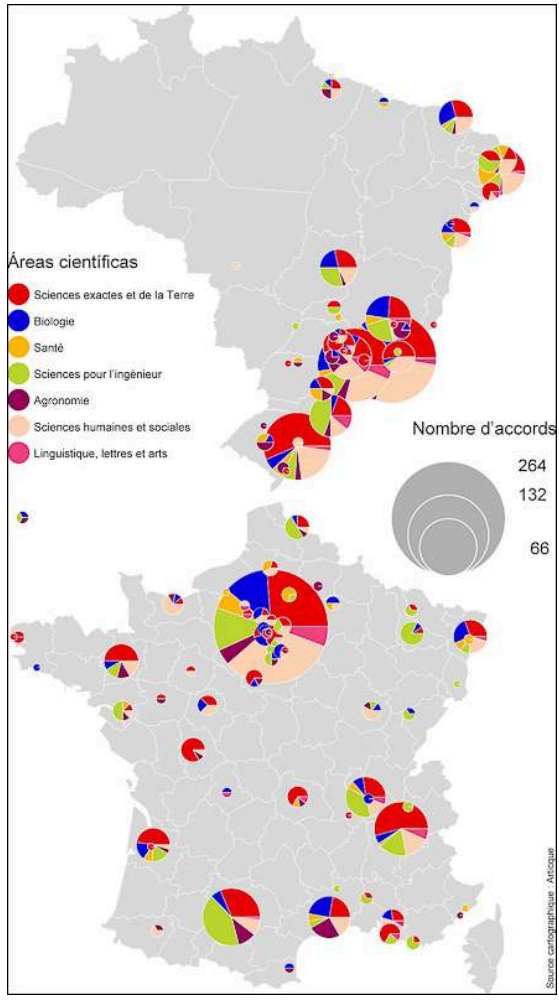

Figure 5 - Prédominance des champs scientifiques par ville

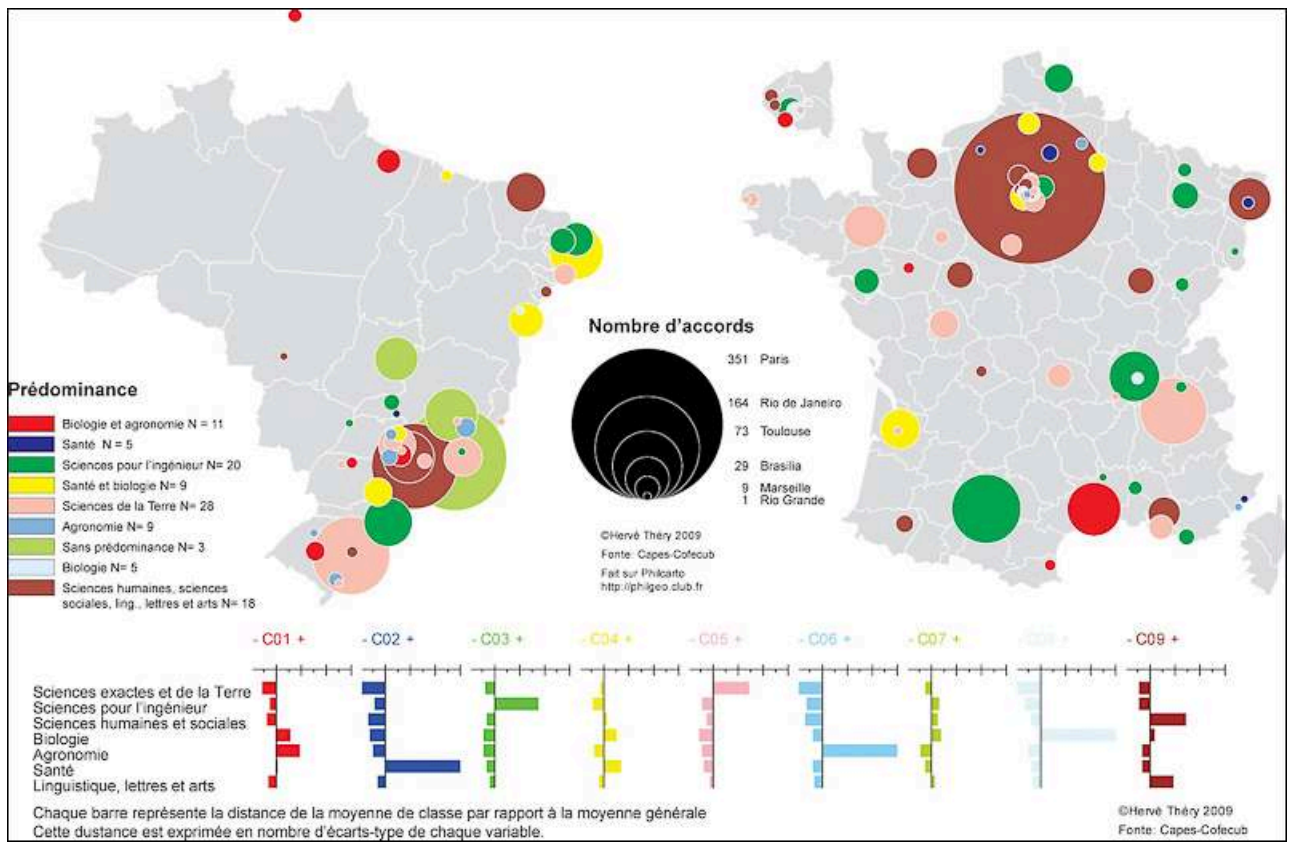

Source : Capes-Cofecub - H. Théry, 2009

13 Une analyse par classification hiérarchique ascendante (figure 5) permet de synthétiser ces «spécialisations » des villes universitaires en se fondant sur profils, les domaines scientifiques où les villes sont au-dessus de la moyenne (barres à droites) ou au-dessous (barres à gauche) : par exemple en sciences agraires Piracicaba et Viçosa, en sciences de 
la Terre Porto Alegre, Grenoble et Rennes, en sciences humaines et sociales Paris et São Paulo, etc.

\section{Les flux principaux}

14 Représenter les collaborations existant entre universités françaises et brésiliennes, dans le cadre du programme Capes-Cofecub, sous forme de cartes de flux fait apparaître un tissu dense (figure 5), au point qu'il faut pour plus de clarté le réduire aux cas les plus nombreux (figure 6). Il apparaît de plus un certain nombre de spécialisations qu'il vaut la peine d'analyser de plus près puisqu'elles révèlent des affinités entre les établissements, ou du moins entre des équipes y travaillant.

Figure 6 - Nombre total d'accords

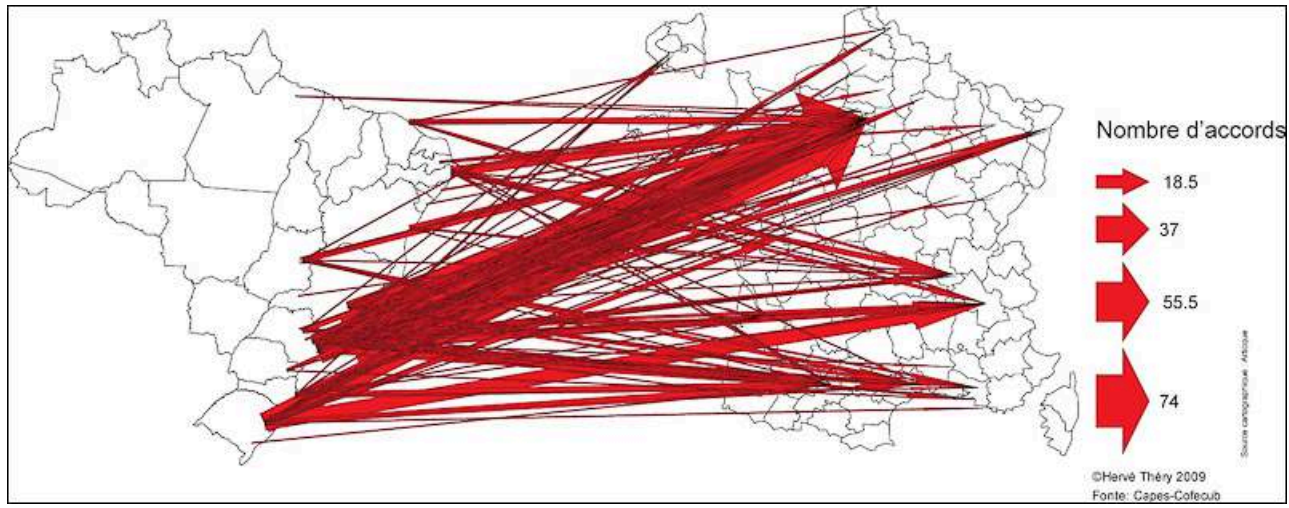

Source : Capes-Cofecub - H. Théry, 2009.

Figure 7 - Plus de trois accords

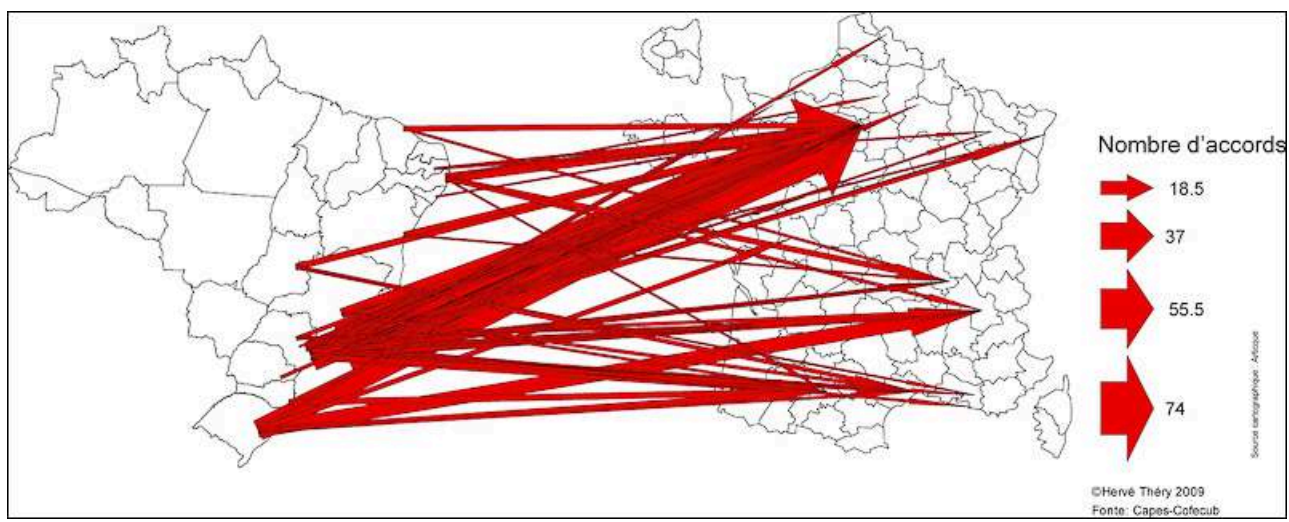

Source : Capes-Cofecub - H.Théry, 2009

Tableau 3 - Principaux accords par ville (plus de 9 accords)

\begin{tabular}{|l|l|l|}
\hline & Ville brésilienne & Ville française \\
\hline 74 & Rio de Janeiro & Paris \\
\hline 42 & Sao Paulo & Paris \\
\hline
\end{tabular}




\begin{tabular}{|l|l|l|}
\hline 29 & Porto Alegre & Paris \\
\hline 20 & Porto Alegre & Grenoble \\
\hline 14 & Recife & Paris \\
\hline 13 & Belo Horizonte & Paris \\
\hline 11 & Rio de Janeiro & Toulouse \\
\hline 11 & São Paulo & Toulouse \\
\hline 10 & São Paulo & Grenoble \\
\hline 9 & Niterói & Paris \\
\hline 9 & Campinas & Paris \\
\hline 9 & Florianópolis & Toulouse \\
\hline 9 & Brasília & Paris \\
\hline
\end{tabular}

15 Les principaux échanges, vus du côté brésilien, concernent les villes de Rio de Janeiro, São Paulo, Porto Alegre, Belo Horizonte et Brasília, les autres villes ayant nettement moins d'échanges avec leurs homologues françaises, au point qu'elles ont pu être regroupées sur la figure 9.

Les projets issus de Rio concernent principalement Paris, comme le montre bien l'épaisse flèche de la figure 7, ce qui n'est pas aussi nettement le cas pour São Paulo. Celle-ci développe aussi des échanges importants avec Grenoble, Montpellier et Toulouse, ce qui équilibre un peu mieux ses échanges avec la France. Dans le cas de Porto Alegre, le poids de Grenoble et de Toulouse est suffisamment fort pour faire jeu presque égal avec Paris, alors que Belo Horizonte reproduit, sur un mode mineur, le style de Rio. Dans les autres villes, le nombre de projets n'est pas suffisant pour que l'on puisse tirer des conclusions claires, mais dans tous les cas de figure c'est Paris, et secondairement Grenoble, qui se détachent. 
Figure 8 - Rio de Janeiro et São Paulo

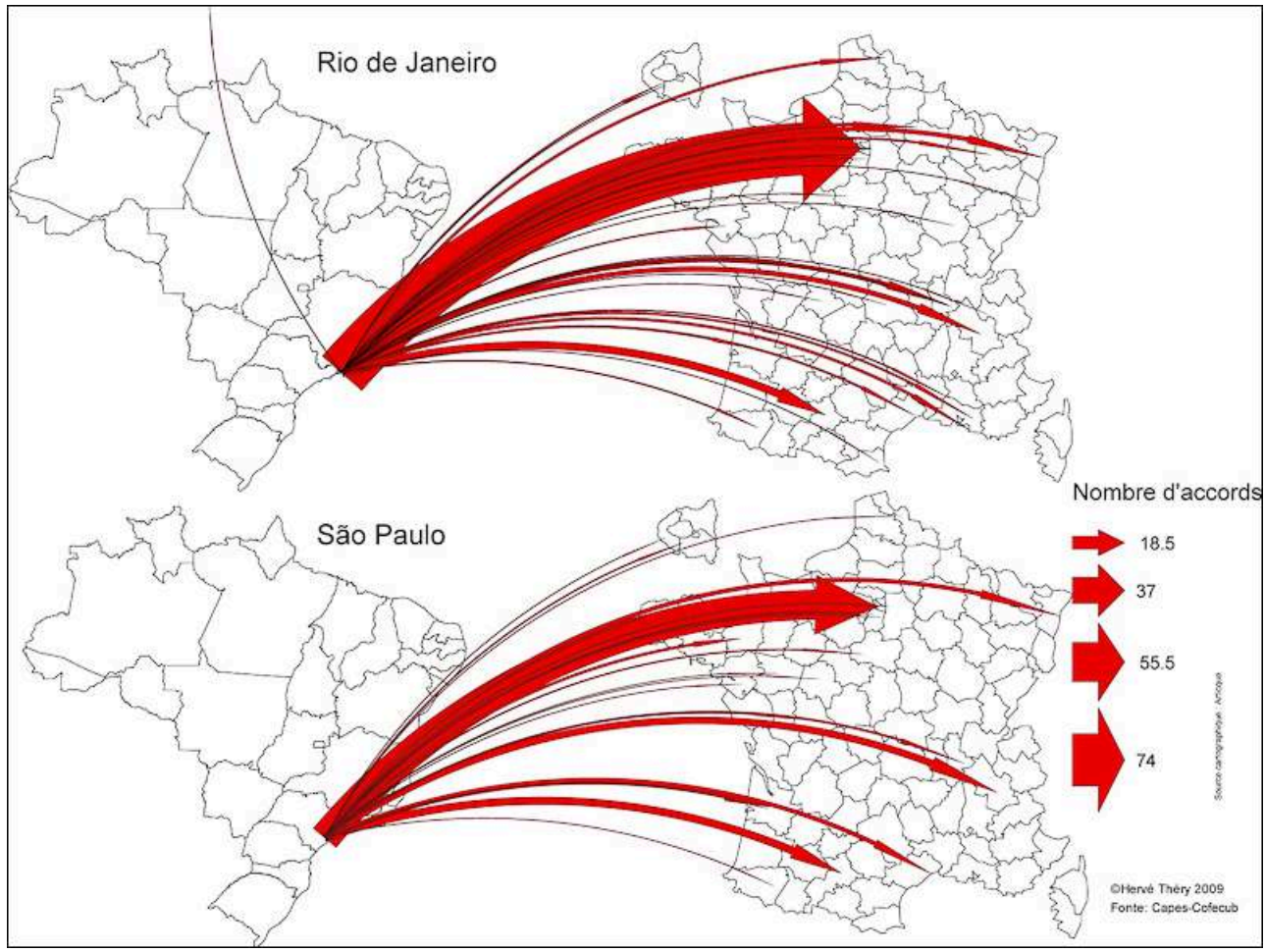

Source : Capes-Cofecub - H. Théry, 2009.

Figure 9 - Porto Alegre et Belo Horizonte

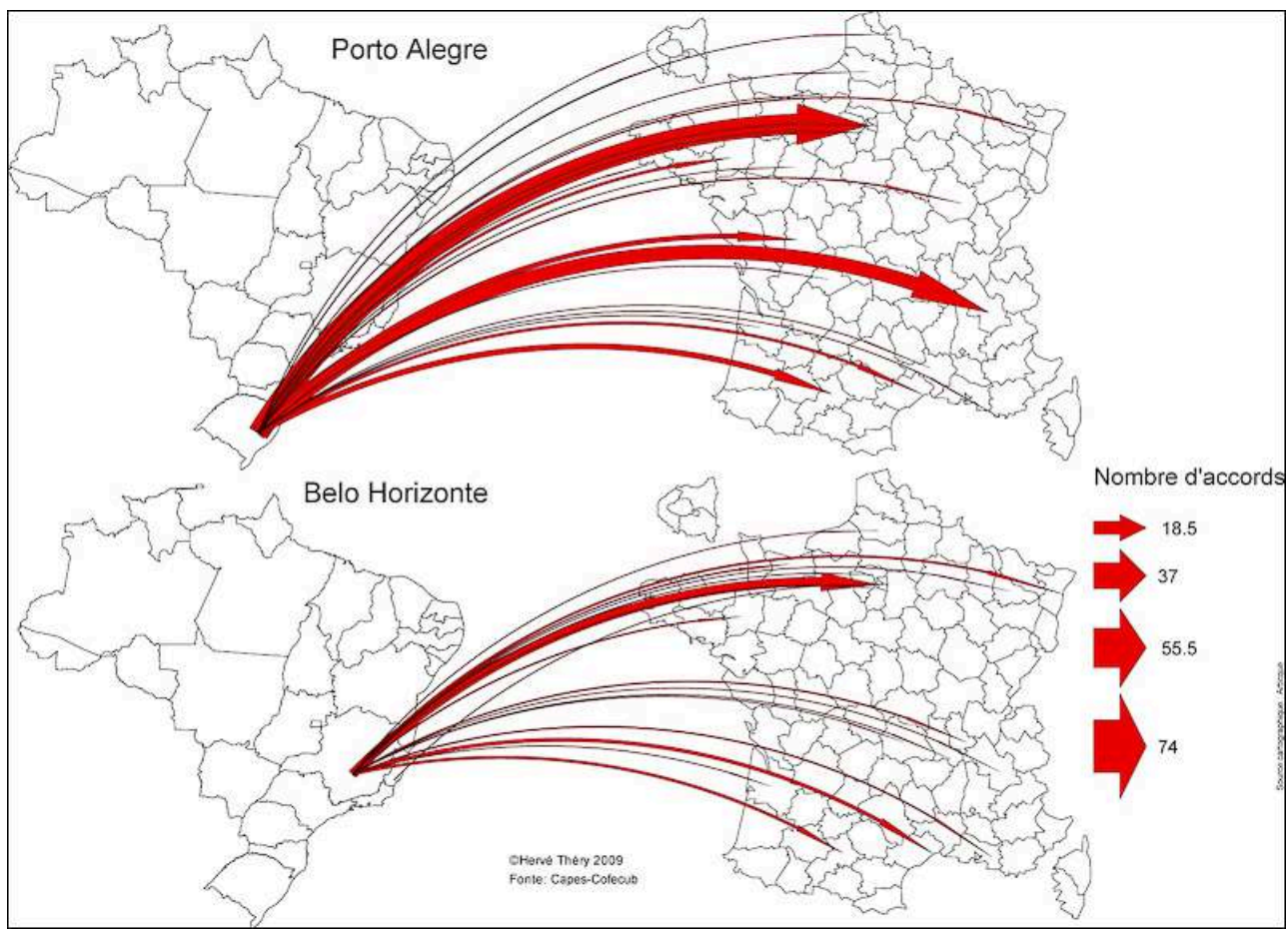

Source : Capes-Cofecub - H. Théry, 2009. 
Figure 10 - Brasília et autres villes

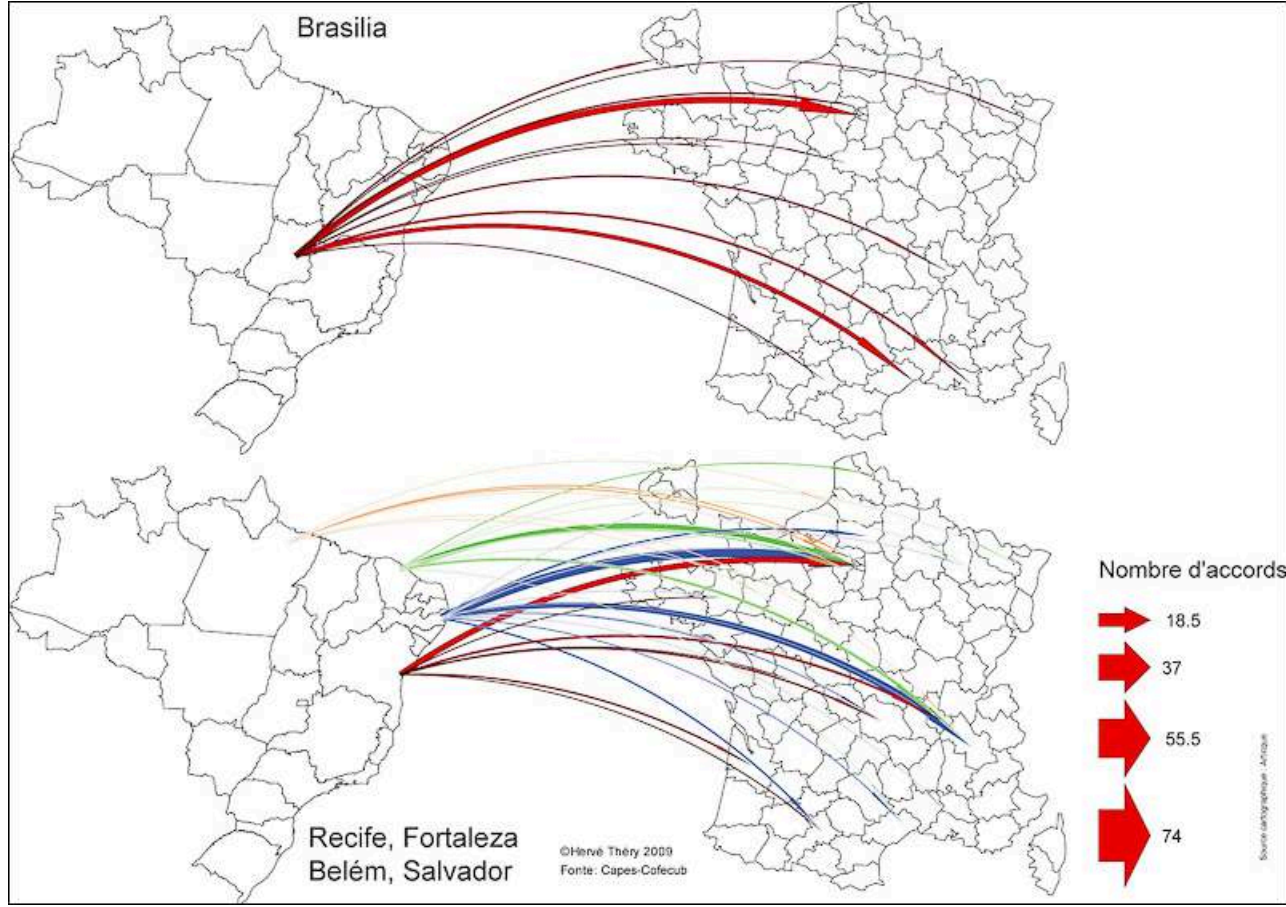

Source : Capes-Cofecub - H. Théry, 2009.

Les projets vus du côté français font évidemment la part belle à Paris, puisque c'est dans cette ville que se situent les partenaires le plus souvent choisis par les universités brésiliennes: les flux qui en sont issus sont nombreux et puissants, ils se dirigent principalement vers le sud et le sud-est du pays, les régions les plus développées sur le plan économique mais aussi sur le plan scientifique. À partir de Toulouse, Grenoble, Montpellier et Rennes les flux de projets concernent également les mêmes régions du pays, de façon plus concentrée encore, la seule exception au nord du Minas Gerais étant Brasilia, la capitale fédérale. Les autres grandes villes universitaires font des choix similaires, privilégiant le Sud et Sudeste au détriment du Nordeste, avec lequel les projets sont relativement peu nombreux, et plus encore le Nord amazonien, qui n'en compte pratiquement aucun. 
Figure 11 - Paris, Toulouse, Grenoble, Montpellier et Rennes

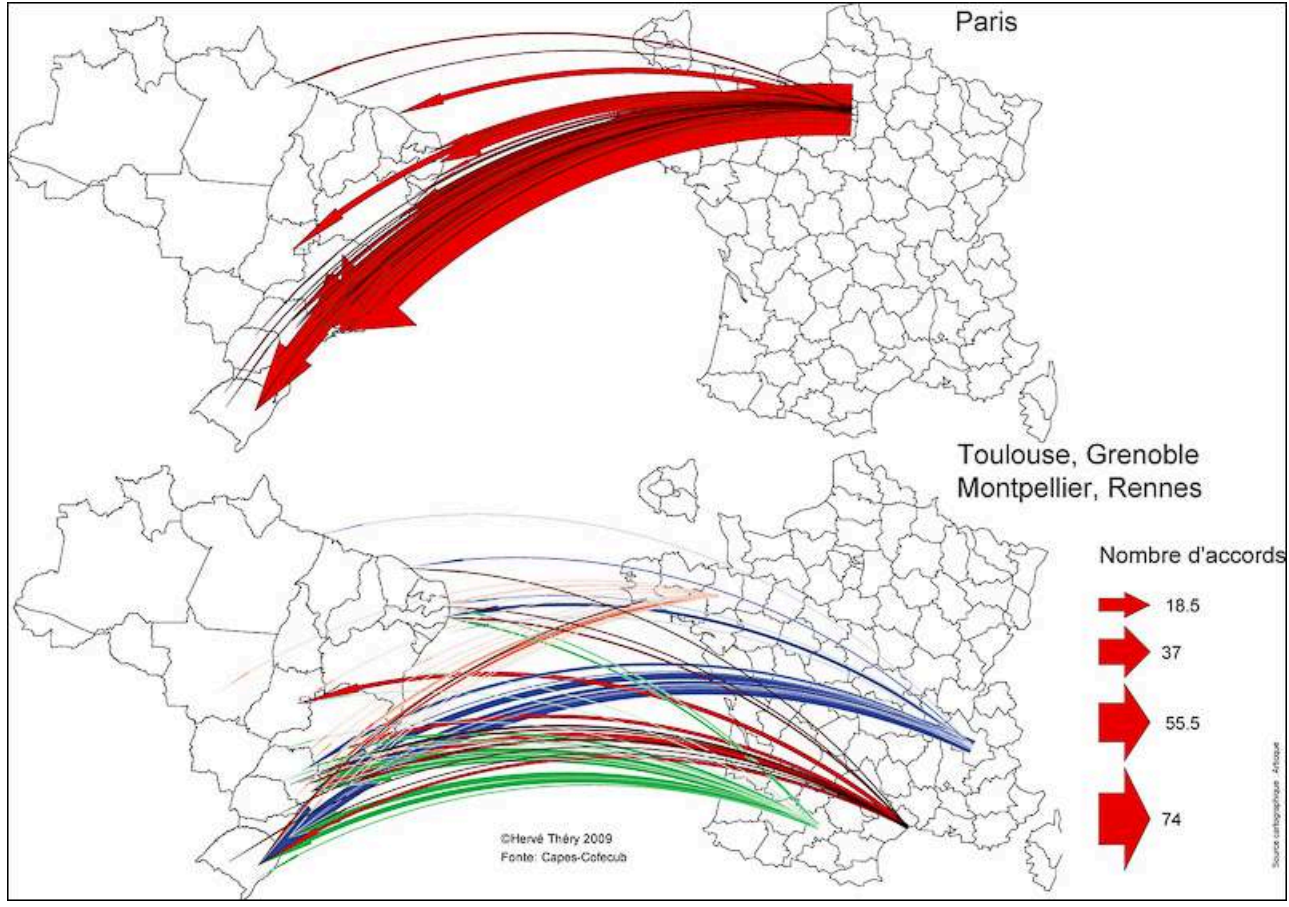

Source : Capes-Cofecub - H. Théry, 2009.

Figure 12 - Autres grandes villes

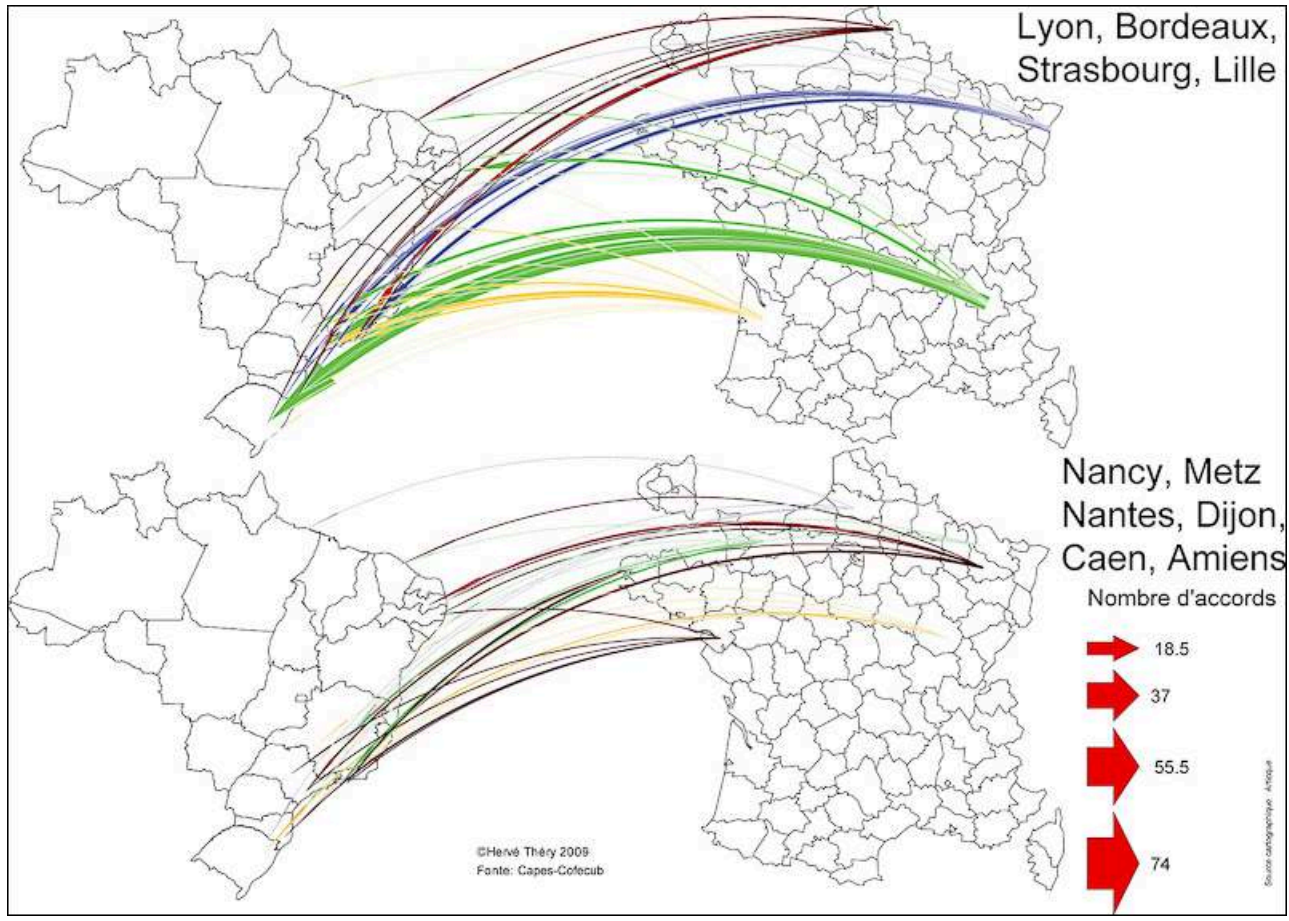

Source : Capes-Cofecub - H. Théry, 2009. 


\section{Le cas de la géographie}

Dans le cas spécifique de la géographie, qui nous concerne plus directement, les centres principaux sont également Paris et la région parisienne du côté français et le triangle des capitales du Sudeste (Rio de Janeiro, São Paulo et Belo Horizonte) du côté brésilien. Trois capitales situées dans les régions plus périphériques du Sud, du Nordeste et du Centre-Ouest, Recife, Porto Alegre et Cuiabá, ont pu monter leurs propres échanges : Recife avec Paris, Cuiabá avec Rennes tandis que Porto Alegre s'est alliée à l'université du Mans, une nouvelle venue sur la carte des échanges franco-brésiliens. Du côté français, la répartition est moins centrée sur la région de la capitale, Strasbourg, Pau et Montpellier y trouvent leur place, ainsi que Rennes et Le Mans, déjà citées. On notera ici l'absence de villes dotées de départements de géographie importants, comme Toulouse ou Grenoble, ce qui indique à l'évidence que les relations avec le Brésil ne sont pas automatiques, mais supposent des initiatives d'équipe et de personnes capables d'établir des ponts avec leurs homologues brésiliens.

Figure 13 - Les accords en géographie

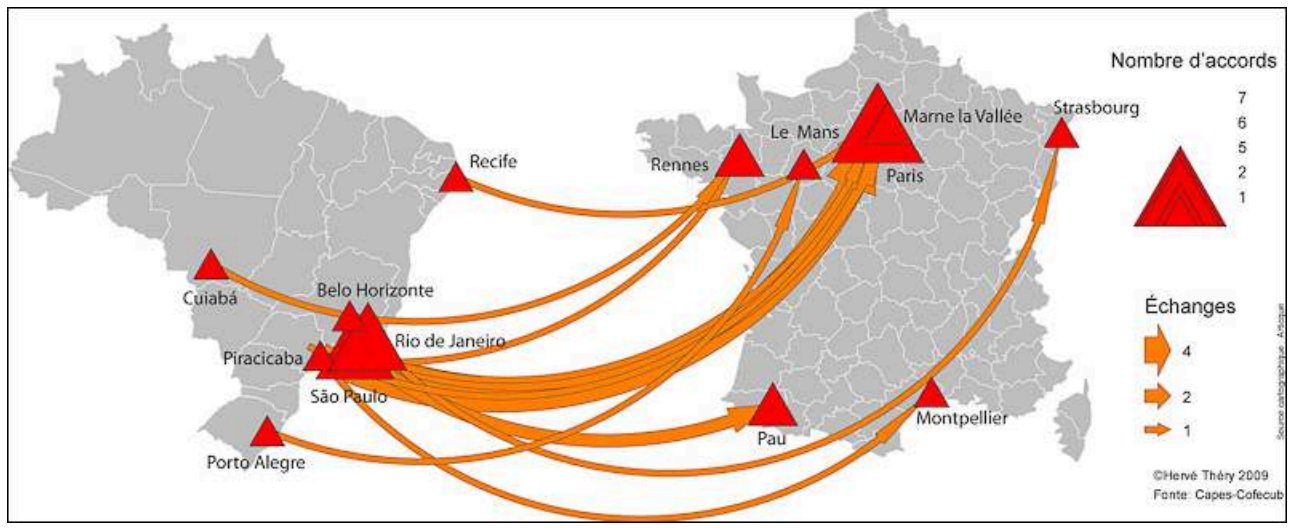

Source : Capes-Cofecub - H.Théry, 2009

19 C'est ce que confirme l'examen de la liste des 16 projets approuvés, en géographie, au fil de ces 30 années. On y voit figurer des personnalités bien connues dans le petit monde des «brésilianistes » français, et leurs homologues brésiliens, qui ne le sont pas moins dans leurs domaines respectifs. Le fait qu'un projet soit porté par J. Pereira de Queiróz et A. Ruellan,, par exemple, a dû peser davantage dans l'évaluation que les mérites respectifs de l'USP et de l'Université de Rennes, si grands soient-ils. On peut en trouver la confirmation dans le fait que le même A. Ruellan obtient ensuite un nouveau projet depuis Montpellier, J. Pereira de Queiróz continuant l'ancien projet, cette fois avec l'Université Paris VII. De la même façon, P.C. da Costa Gomes et V. Berdoulay ont pu renouveler leur accord, sur un thème voisin du premier, tout comme G. Cocco et $A$. Bourdin. On sait bien que le fondement d'un bon échange Capes-Cofecub, qui dure et se renouvelle, est bien l'accord entre des personnes qui se connaissent et se font confiance, savent pouvoir compter l'une sur l'autre au moment de monter équipes et programme de recherche. 
Tableau 4 - Accords en géographie

\begin{tabular}{|c|c|c|c|c|c|c|}
\hline & $\begin{array}{l}\text { Coordinateur } \\
\text { brésilien }\end{array}$ & $\begin{array}{l}\text { Institution } \\
\text { brésilienne }\end{array}$ & $\begin{array}{l}\text { Ville } \\
\text { brésilienne }\end{array}$ & $\begin{array}{l}\text { Coordinateur } \\
\text { français }\end{array}$ & $\begin{array}{l}\text { Institution } \\
\text { française }\end{array}$ & $\begin{array}{l}\text { Ville } \\
\text { française }\end{array}$ \\
\hline $035 / 87$ & $\begin{array}{l}\text { J. Pereira de } \\
\text { Queiróz }\end{array}$ & USP & São Paulo & A. Ruellan & U. Rennes & Rennes \\
\hline $125 / 91$ & A. Saadi & UFMG & $\begin{array}{l}\text { Belo } \\
\text { Horizonte }\end{array}$ & H. Vogt & ULP & Strasbourg \\
\hline $083 / 87 / 92$ & N. Lacerda & UFPE & Recife & C. Gros & U. Paris III & Paris \\
\hline $151 / 94$ & $\begin{array}{l}\text { S. Simões de } \\
\text { Castro }\end{array}$ & USP & São Paulo & A. Ruellan & CNEARC & Montpellier \\
\hline $005 \mathrm{~N} / 96$ & $\begin{array}{l}\text { G. Tomasini } \\
\text { Maitelli }\end{array}$ & UFMT & Cuiabá & R. Bariou & Rennes II & Rennes \\
\hline $190 / 96$ & $\begin{array}{l}\text { C. Egler / I. de } \\
\text { Castro }\end{array}$ & UFRJ & $\begin{array}{l}\text { Rio de } \\
\text { Janeiro }\end{array}$ & M. Droulers & U. Paris III & Paris \\
\hline $333 / 00 / 02$ & $\begin{array}{l}\text { P.C. da Costa } \\
\text { Gomes }\end{array}$ & UFRJ & $\begin{array}{ll}\text { Rio } & \text { de } \\
\text { Janeiro } & \end{array}$ & V. Berdoulay & U. de Pau & $\mathrm{Pau}$ \\
\hline 409/02/04 & $\begin{array}{l}\text { A. I. Geraiges } \\
\text { Lemos }\end{array}$ & USP & São Paulo & H. Théry & ENS & Paris \\
\hline $412 / 03 / 05$ & $\begin{array}{l}\text { J. Pereira de } \\
\text { Queiróz }\end{array}$ & USP & São Paulo & M. Fort & U. Paris 7 & Paris \\
\hline $429 / 03 / 05$ & G. Cocco & UFRJ & $\begin{array}{ll}\text { Rio } & \text { de } \\
\text { Janeiro } & \end{array}$ & A. Bourdin & UMV & $\begin{array}{ll}\text { Marne } & \text { la } \\
\text { Vallée } & \end{array}$ \\
\hline $483 / 05$ & $\begin{array}{l}\text { Célia Regina } \\
\text { Montes }\end{array}$ & USP/Esalq & Piracicaba & G. Calas & U. Paris VI & Paris \\
\hline 2006-18 & G. Cocco & UFRJ & $\begin{array}{ll}\text { Rio } & \text { de } \\
\text { Janeiro } & \end{array}$ & A. Bourdin & UMV & $\begin{array}{ll}\text { Marne la } \\
\text { Vallée }\end{array}$ \\
\hline $2007-114$ & $\begin{array}{l}\text { P.C. da Costa } \\
\text { Gomes }\end{array}$ & UFRJ & $\begin{array}{ll}\text { Rio } & \text { de } \\
\text { Janeiro }\end{array}$ & V. Berdoulay & U Pau & $\mathrm{Pau}$ \\
\hline $2007-116$ & R. Verdum & UFRGS & $\begin{array}{l}\text { Porto } \\
\text { Alegre }\end{array}$ & J. Cobornnois & U du Maine & Le Mans \\
\hline 2008-71 & $\begin{array}{l}\text { A.F. } \\
\text { Alessandri } \\
\text { Carlos }\end{array}$ & USP & São Paulo & H. Théry & U. Paris III & Paris \\
\hline
\end{tabular}


Tableau 5 - Thèmes des accords en géographie

\begin{tabular}{|c|c|}
\hline Numéro & Titre \\
\hline $035 / 87$ & Pédologie \\
\hline $125 / 91$ & Géomorphologie, les marges sud et est du craton do São Francisco \\
\hline 083/87/92 & Pouvoir, territoire et technologie \\
\hline $151 / 94$ & $\begin{array}{l}\text { Morphologie des systèmes pédologiques tropicaux : relations entre fonctionnement et } \\
\text { fertilités }\end{array}$ \\
\hline $005 \mathrm{~N} / 96$ & Écosystèmes et organisation de l'espace en Amazonie \\
\hline $190 / 96$ & Géographie, gestion du territoire et développement durable \\
\hline $333 / 00 / 02$ & Les lieux du politique : espaces publics et espaces de gestion \\
\hline $409 / 02 / 04$ & $\begin{array}{l}\text { Les défis de la mondialisation et les dynamiques régionales au Brésil et en Amérique du } \\
\text { Sud }\end{array}$ \\
\hline $412 / 03 / 05$ & $\begin{array}{l}\text { Processus physiques, hydriques et biogéochimiques du Pantanal da Nhecolândia et ses } \\
\text { représentations spatiales }\end{array}$ \\
\hline $429 / 03 / 05$ & La ville, territoire productif : réseaux, coopérations et gouvernances \\
\hline $483 / 05$ & Podzolisation des latérites du haut bassin amazonien \\
\hline $2006-18$ & La ville, territoire productif : réseaux, coopérations et gouvernances \\
\hline 2006-3 & $\begin{array}{l}\text { Processus physiques, hydriques et biogéochimiques du Pantanal da Nhecolândia et ses } \\
\text { représentations spatiales }\end{array}$ \\
\hline $2007-114$ & Les scénarios de l'urbanité : images, espaces et identités \\
\hline $2007-116$ & Arénisation et gestion des ressources hydriques dans le bassin du Rio Ibicuí \\
\hline $2009-71$ & $\begin{array}{l}\text { Différenciation et dynamique spatiale: échelles, processus et instruments } \\
\text { d'observation }\end{array}$ \\
\hline
\end{tabular}

Dans ce cas comme dans bien d'autres, la carte permet de déceler des configurations spatiales que les données brutes, sous forme de listings ou de tableaux de chiffres, ne permettaient pas de voir. Outre l'effet évident des hiérarchies urbaines - les villes les plus peuplées sont aussi les grands centres universitaires - on voit apparaitre des spécialisations qui renvoient à des traditions d'excellence dans tel ou tel domaine, elles-mêmes patiemment construites et entretenues par des universitaires et leurs organismes de financement. Mais la réussite d'un accord, de l'initiative initiale au passage des filtres rigoureux de la sélection binationale, est la résultante des efforts de petits groupes - en l'occurrence celle des équipes candidates - voire même de l'action individuelle de personnes motrices. 


\section{BIBLIOGRAPHIE}

Ano da França http ://anodafrancanobrasil.cultura.gov.br/fr/2009/05/25/seminariocomemora-30-anos-de-cooperacao-franco-brasileira-com-resultados-positivos/ consulté le $4 / 2 / 2011$

Capes http ://www.capes.gov.br/sobre-a-capes/historia-e-missao et http ://www.capes.gov.br/ cooperacao-internacional/franca/cofecub, consultés le 4/2/2011

Carelli M., Théry H., Zantman A., 1987. France-Brésil : bilan pour une relance, Entente, Paris, 274 p.

Cofecub http ://www.egide.asso.fr/jahia/Jahia/lang/fr/accueil/appels/cofecub, consulté le $4 / 2 / 2011$

Hamburger A. I. (org.), 1986. A ciência nas relações Brasil-França, 1850-1950. Edusp, São Paulo, 359 p.

Lefebvre J.P., 1990, Les professeurs français des missions universitaires au Brésil (1934-1944).

Cahiers du Brésil Contemporain, 1990, http ://www.revues.msh-paris.fr/vernumpub/8-

J.P \%20Lefebvre.pdf

Martinière G., 1982. Aspects de la coopération franco-brésilienne, Collection Brasilia, Paris, Éditions de la Maison des sciences de l'homme.

Nunes, B. Ferreira, 2006. O sistema de C T no Brasil e a cooperação internacional :notas sobre a experiência Capes/Cofecub. R B P G, Brasília, v. 3, n. 6, p. 234-253, dez. 2006, http :// www2.capes.gov.br/rbpg/images/stories/downloads/RBPG/Vol.3_6_dez2006_/ EXP_Artigo1_n6.pdf

Théry, Hervé, 2009. Uma geografia da cooperação universitária França-Brasil, analise dos acordos acadêmicos Capes-Cofecub. Revista da Anpege, n 5, p. 140-156, http ://anpege.org.br/revista/ ojs-2.2.2/index.php/anpege08/article/view/34

\section{NOTES}

1. Sciences du Vivant, Physique, Chimie, Sciences de l'Ingénieur, Sciences Humaines et Sociales, Médecine-Santé, Terre et Univers, Mathématiques et Sciences et Techniques de l'Information et de la Communication.

2. Dont on peut remarquer avec intérêt, au titre des comparaisons culturelles entre les deux pays, qu'elles sont respectivement appelées « camembert » en France et « pizza » au Brésil...

\section{RÉSUMÉS}

L'analyse et la cartographie des 750 accords de coopération universitaire entre la France et le Brésil fait apparaitre, au-delà de l'effet évident des hiérarchies urbaines (les villes les plus peuplées sont aussi les grands centres universitaires), des spécialisations qui renvoient à des traditions locales et la résultante des réseaux des équipes candidates. 
The analysis and mapping of the 750 agreements for academic cooperation between France and Brazil shows, beyond the obvious effect of urban hierarchies (the most populous cities are also the major academic centers), specializations wich refer to local traditions and the result of networking made by the candidate teams.

INDEX

Mots-clés : Brésil, Capes-Cofecub, coopération universitaire, sciences, Universités

Keywords : academic cooperation, Brazil, Capes-Cofecub, sciences, Universities

\section{AUTEUR}

\section{HERVÉ THÉRY}

Hervé Théry, hthery@aol.com, est Directeur de recherche au CNRS-Creda, UMR 7227, Professor convidado na USP. Il a publié récemment :

- Théry H., 2010. Le Brésil et le Monde. In Les relations internationales du Brésil, les chemins de la puissance, Denis Rolland et Antônio Carlos Lessa (coord.), L'Harmattan, ISBN 978-2-296-13543-7, p 61-72.

- Théry H., 2005. Le Brésil, Armand Colin, 5e édition, 288 p.

- Théry H. et Aparecida de Mello N, 2003. Atlas du Brésil, CNRS Libergéo - La Documentation

Française, 304 p. (traduction Atlas do Brasil, Disparidades e dinâmicas do território, EDUSP, São Paulo, 2005 et 2008). 